\title{
Magnetic behavior of poly(3-methylthiophene): Metamagnetism and room-temperature weak ferromagnetism
}

\author{
O. R. Nascimento \\ Instituto de Física de S. Carlos, Universidade de São Paulo, Caixa Postal 369, CEP 13560-970, S. Carlos, São Paulo, Brazil \\ A. J. A. de Oliveira \\ Departamento de Física, Universidade Federal de São Carlos, Caixa Postal 676, CEP 13560-970, São Carlos, São Paulo, Brazil
}

A. A. Correa, L. O. S. Bulhões, and E. C. Pereira

Departamento de Química, Universidade Federal de São Carlos, Caixa Postal 676, CEP 13560-970, São Carlos, São Paulo, Brazil

V. M. Souza and L. Walmsley

Departamento de Física, Instituto de Geociências e Ciências Exatas, Universidade Estadual Paulista, Caixa Postal 178, CEP 13500-970, Rio Claro, São Paulo, Brazil

(Received 13 December 2002; published 28 April 2003)

\begin{abstract}
A weak ferromagnetic phase is shown in pressed pellets of partially doped poly(3-methylthiophene) (P3MT) in the whole range from 1.8 to $300 \mathrm{~K}$ in magnetic measurements. Thermoremanence data have been used to estimate the suppression of this phase to be around $815 \mathrm{~K}$. We also show that instead of the classical antiferromagnetism for the first-order interaction that gives weak ferromagnetism as a second-order effect, metamagnetic behavior is observed. $X$-band electron spin resonance (ESR) measurements and magnetization measurements allowed us to estimate that $8.1 \%$ of the total number of spins contributes to the weak ferromagnetism at room temperature. The doping level obtained from the ESR data is in good agreement with that estimated from electron dispersive spectroscopy measurements.
\end{abstract}

DOI: $10.1103 /$ PhysRevB.67.144422

PACS number(s): 75.50.Kj, 76.30.-v, 82.35.Cd

\section{INTRODUCTION}

Since the discovery of conductors polymers in the 1970 's 1,2 this area has quickly increased because of the large spectrum of applications and many physical phenomena related with these materials. In conducting polymers the magnetic moments are created upon doping. When a conjugated polymer with a nondegenerate ground state is doped with an electron or hole, a polaron, which is an ion radical selftrapped in a local lattice distortion, is produced. A polaron has spin $1 / 2$ and charge $e$ (for hole doping). If the polymer is heavily doped, two polarons will form a bipolaron with zero spin and charge $2 e$.

The possibility of ferromagnetic behavior in conducting polymers has been theoretically addressed ${ }^{3,4}$ and evidence of this interaction has been reported. ${ }^{5,6}$ In a recent paper Rajca et $a .^{7}$ have also reported magnetic ordering at low temperatures in an organic polymer.

In recent articles ${ }^{8,9}$ we have reported the direct observation of a weak ferromagnetic phase in pressed pellets of $\mathrm{ClO}_{4}^{-}$partially doped poly(3-methylthiophene). This magnetic behavior was observed in the full range from 1.8 to 300 $\mathrm{K}$ in measurements performed using a superconducting quantum interference device (SQUID) magnetometer. We have shown that the magnetization depends on the thermal history, pressure used to make the pellets, doping level, and water content of the solvent. The intrinsic character of this behavior was shown from an atomic absorption analysis that discarded contamination. ${ }^{8}$ This result was confirmed from measurements performed in a sample immediately after synthesis (ferromagnetic behavior) and five months after keep- ing the same sample in air at room temperature (diamagnetic behavior). ${ }^{9}$

We have attributed the ferromagnetic behavior to the polarons formed in the polymeric chains, which we suppose experience a superexchange interaction via the dopant anions. The partially crystalline low symmetry system allows a weak ferromagnetic behavior to arise because of a Dzialoshinski-Morya interaction among canted spins. ${ }^{10}$

In this article we show that instead of the classical antiferromagnetism for the first-order interaction that gives weak ferromagnetism as a second-order effect, metamagnetic behavior is observed. ${ }^{11}$ We present magnetization measurements that show in addition to the weak ferromagnentic behavior, evidence of this metamagnetism and that it is in good agreement with the spin-1/2 polaronic character. Thermoremanence data is presented for the characterization of the ferromagnetic behavior.The magnetic interaction can also be observed from $X$-band electron spin resonance (ESR) measured for the same thermal history and range of temperatures and magnetic fields (1-5 kOe) of the magnetization measurements. Finally, from the comparison of the ESR and magnetic measurements we present a better understanding of the complex magnetic behavior of the system.

\section{EXPERIMENTAL DETAILS}

Poly(3-methylthiophene) (P3MT) was synthesized at $25^{\circ} \mathrm{C}$ using a constant potential of $1.48 \mathrm{~V}$ (referred to as a quasireference silver electrode) in acetonitrile with the addition of $200 \mathrm{ppm}$ of water, $0.1 \mathrm{M}$ methylthiophene, and $0.1 \mathrm{M}$ $\mathrm{LiClO}_{4}$. The synthesis was performed in a dry box filled with 

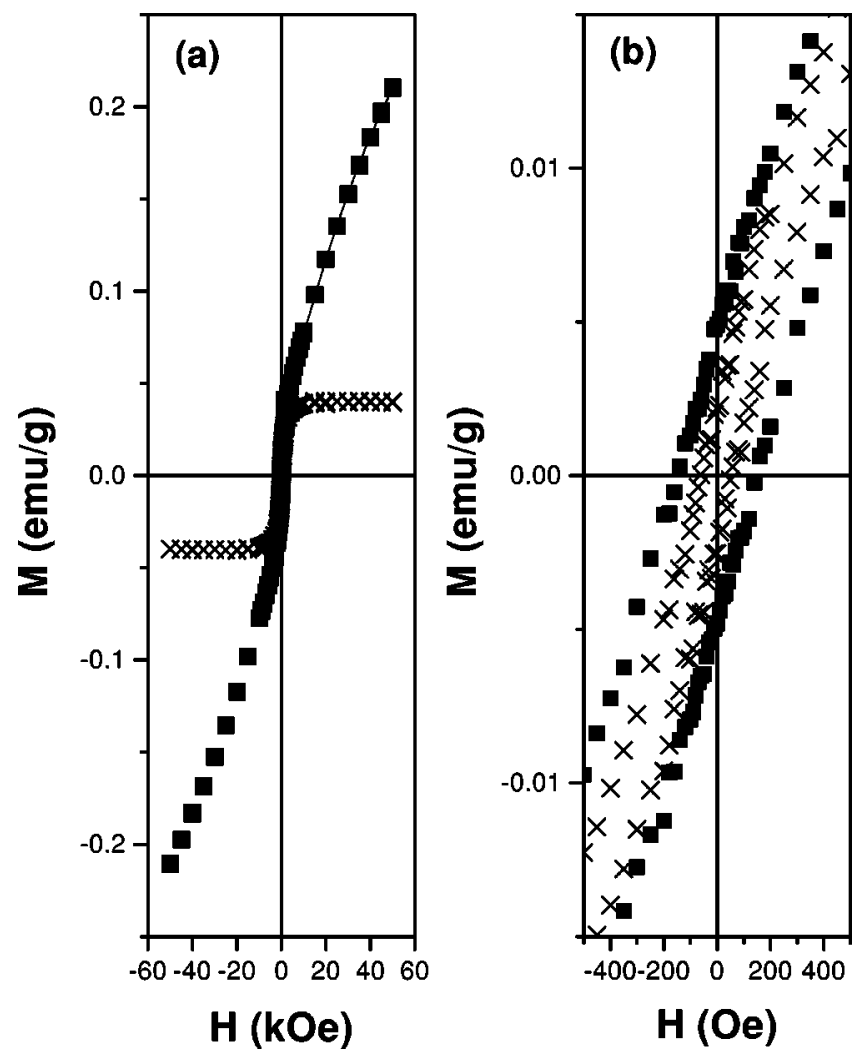

FIG. 1. $M$ versus $H$ curves of sample A at 5 (squares) and $300 \mathrm{~K}$ (crosses). Solid line: fitting with the expression $M(H)=M_{1}$ $+M_{2} \tanh (\alpha H)$ for $H \geqslant 5$ kOe. Details of the ferromagnetic hysteresis.

argon. Electrochemical deposition with the total charge fixed at $100 \mathrm{C}$, resulted in a powdered sample on the Pt electrode. After the synthesis, the open circuit potential of the cell $V_{o c}$ was $0.8 \mathrm{~V}$ indicating that the polymer was in the oxidized state. The solution was changed twice to remove the soluble oligomers and the polymer was then partially reduced in a $0.1 \mathrm{M}$ acetonitrile solution with $0.1 \mathrm{M} \mathrm{LiClO}_{4}$ where a final value of $V_{o c}$ was $0.35 \mathrm{~V}$. Pellets were prepared using a pressure of $250 \mathrm{bar}$ in an isostatic chamber, where the powder was enclosed in a capsule of silicone ribbon and the pressure was applied in all but one direction. These particular conditions allowed us to obtain the highest values of remanent magnetization and coercive field at room temperature.

Magnetic measurements were performed using a Quantum Design SQUID magnetometer model MPMS-5S. After the measurements a small piece of the sample (sample A) was encapsulated in a quartz tube containing argon. The sample was placed in a $\mathrm{TE}_{102} X$-band ESR cavity at $4.2 \mathrm{~K}$ and the ESR spectra were recorded raising the temperature from 4.2 to $286 \mathrm{~K}$ (a Varian E-109 spectrometer was used with an ESR Oxford cryostat). We did not observe any skindepth effects in the spectra. At $4.2 \mathrm{~K}$ the ESR signal was very intense and a small microwave power $(P=0.16 \mathrm{~mW})$ was used to detect it in the full range of temperatures.

dc electrical measurements were performed in a similar sample (sample B) using a Keithley 617 electrometer/source

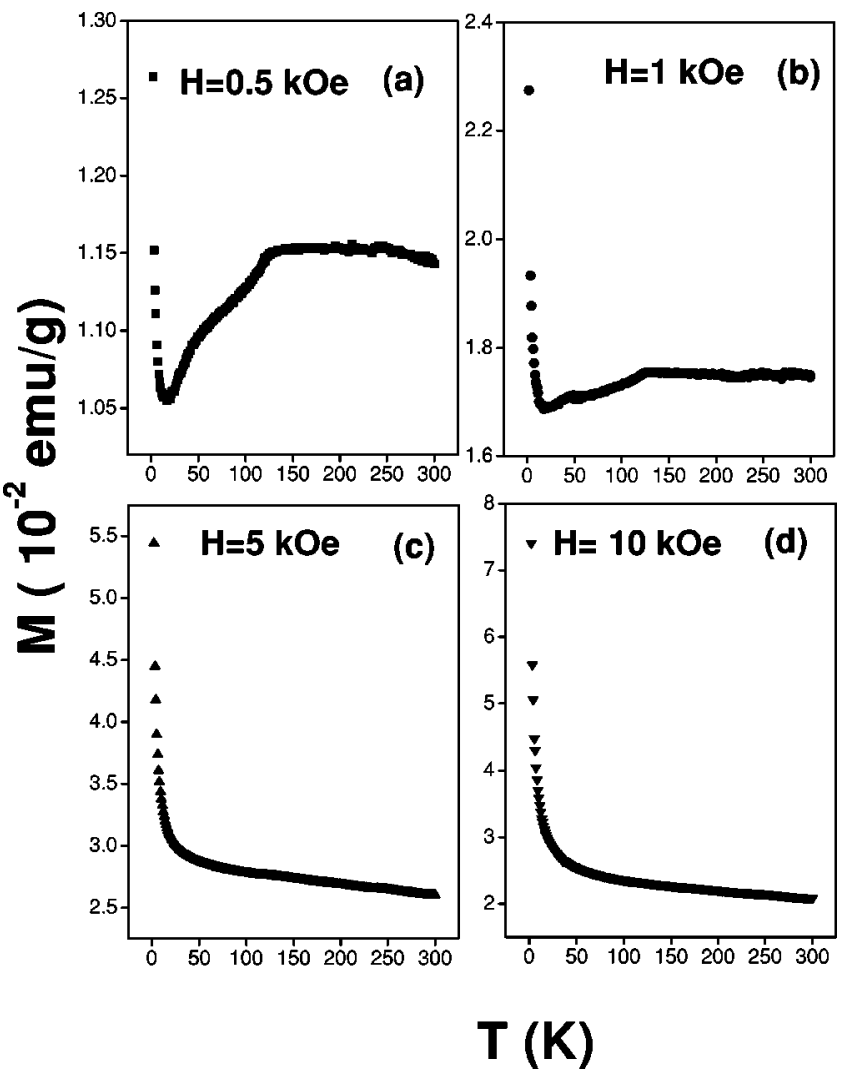

FIG. 2. Zero-field cooling dc magnetic susceptibility of sample A measured with a SQUID magnetometer. (a) $H=500 \mathrm{Oe}$; (b) $H$ $=1 \mathrm{kOe}$; (c) $H=5 \mathrm{kOe}$; (d) $H=10 \mathrm{kOe}$.

with the sample placed in a Janis model CCS- 150 cryostat. A constant voltage of $2 \mathrm{~V}$ was applied to the sample placed in the cryostat.

The other three samples, prepared using the same procedure, were used to perform magnetic thermoremanence measurements (sample C), low field (10 Oe) zero field cooling measurements (sample D), and electron dispersive spectroscopy (EDS) measurements (sample E).

\section{RESULTS AND DISCUSSION}

Figure 1(a) shows the magnetization as a function of applied magnetic field ( $M$ versus $H$ ) curves for sample A at 5 $\mathrm{K}$ (squares) and $300 \mathrm{~K}$ (crosses). At $5 \mathrm{~K}$ we can observe the metamagnetic behavior in addition to the ferromagnetic behavior. At $300 \mathrm{~K}$ we can see only the ferromagnetic behavior because of the small value of the paramagnetic (metamagnetic) contribution at high temperatures. In these measurements the diamagnetic contribution was subtracted. In the original $M$ versus $H$ curve at $300 \mathrm{~K}$, there is a diamagnetic contribution observed above $3 \mathrm{kOe}$ and that shows linear behavior above around $5 \mathrm{kOe}$. The diamagnetic susceptibility was obtained from the linear fit $\left(\chi_{D}=-1.424\right.$ $\times 10^{-6} \mathrm{emu} \mathrm{g}^{-1} \mathrm{Oe}^{-1}$ ), assuming it is independent of the temperature. In Fig. 1(b) the details of the ferromagnetic hysteresis are shown. At $5 \mathrm{~K}$ the remanent magnetization $M_{r}$ $=5 \times 10^{-3} \mathrm{emu} / \mathrm{g}$ and the coercive field $H_{c}=130 \mathrm{Oe}$. At $300 \mathrm{~K}, M_{r}=1 \times 10^{-3} \mathrm{emu} / \mathrm{g}$ and $H_{c}=90 \mathrm{Oe}$. 


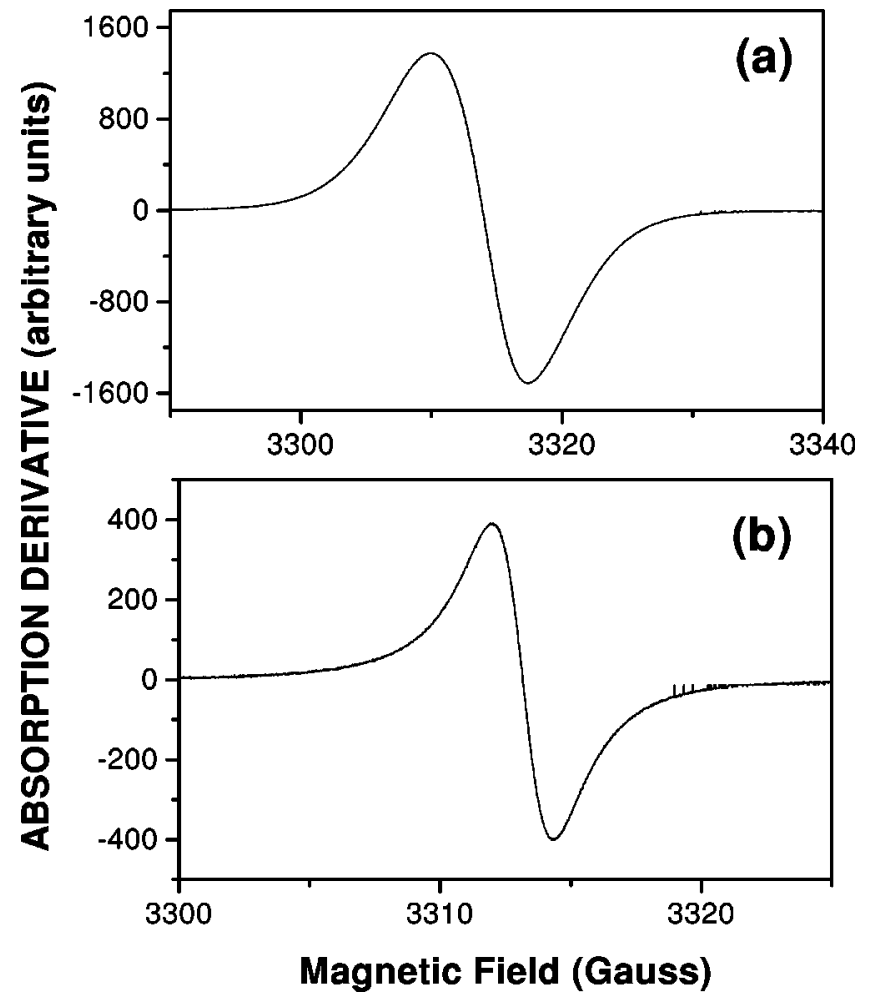

FIG. 3. (a) ESR spectra of sample A at $4.2 \mathrm{~K}$. (b) The same as (a) at $286 \mathrm{~K}$.

For the $5 \mathrm{~K}$ magnetization data of Fig. 1(a) we have performed a fitting (solid line) for fields above $5 \mathrm{kOe}$, using

$$
M(H)=M_{1}+M_{2} \tanh (\alpha H)
$$

The fitted values of $M_{1}=0.0338 \mathrm{emu} / \mathrm{g}, \quad M_{2}$ $=0.256 \mathrm{emu} / \mathrm{g}$, and $\alpha=1.68 \times 10^{-5} \mathrm{Oe}^{-1}$ were found.

From the magnetic behavior observed in Fig. 1 we cannot expect a Curie-Weiss behavior for the magnetization versus temperature data. Figure 2 shows the magnetization measurement as a function of temperature in the range 1.8 to 300 $\mathrm{K}$ with different values of magnetic field: (a) $H=500 \mathrm{Oe}$, (b) $H=1 \mathrm{kOe}$ (c) $H=5 \mathrm{kOe}$, and (d) $H=10 \mathrm{kOe}$, for sample A. The sample was rapidly cooled from room temperature without the application of a field (zero-field cooling procedure) and the measurement was performed raising the temperature at a ratio of $2 \mathrm{~K} / \mathrm{min}$. The metamagnetic character is evident from the change from antiferromagnetic to paramagnetic behavior with the increase of magnetic field.

Figure 3 shows the ESR spectra for sample A (a) at $4.2 \mathrm{~K}$ and (b) at $286 \mathrm{~K}$. For the $4.2 \mathrm{~K}$ data a Gaussian-like line is observed and for the $286 \mathrm{~K}$ data a Lorentzian-like one is seen. This change in line shape starts around $20 \mathrm{~K}$. According to Bencini and Gatteschi, ${ }^{12}$ the Gaussian line is due to slow exchange and Lorentzian lines to fast exchange. In this way the change from Gaussian-like to Lorentzian-like that starts to be observed around $20 \mathrm{~K}$ can be associated to the motion of spins. As expected for a dominant spin-spin interaction, the linewidth increases with the decrease of temperature.

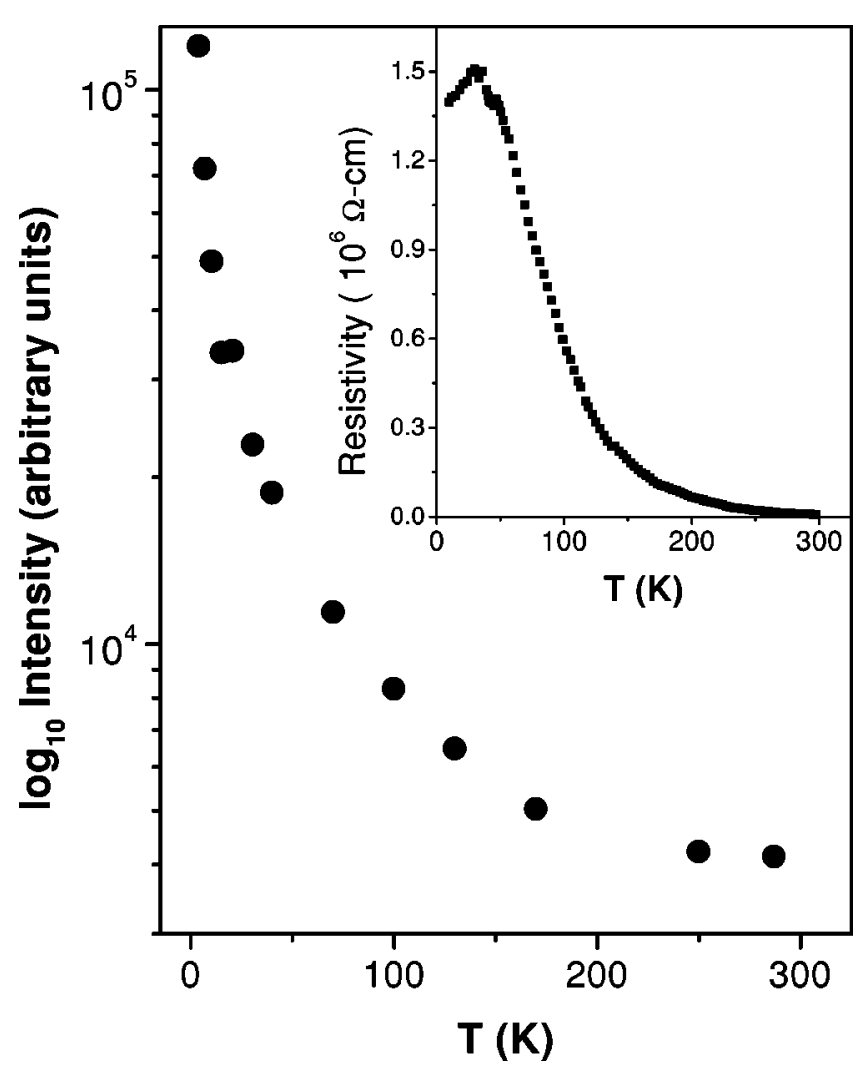

FIG. 4. Intensity of the ESR line versus temperature shown in a logarithmic scale. Inset: Resistivity as a function of temperature for sample B.

Figure 4 shows the ESR intensity in arbitrary units in a logarithmic scale. It was obtained by double integration of the absorption derivative line. In the inset the resistivity versus temperature data for a similar sample (sample B) is shown. The data was obtained raising the temperature from 10 to $300 \mathrm{~K}$ at a rate of $3 \mathrm{~K} / \mathrm{min}$. We can observe high resistivity values in the whole range of temperature that confirms the absence of skin depth effects. In spite of the large values of resistivity, a small increase in the resistivity with temperature was observed followed by a maximum around $20 \mathrm{~K}$ and a decrease in resistivity with the increase of temperature. The data resembles the behavior of a small polaron at low temperatures. ${ }^{13}$ The resistivity data is in reasonable agreement with the change from Gaussian-like to Lorentzian-like behavior observed in the ESR line around 20 $\mathrm{K}$. In attaining the semiconducting state the mobile polarons average out the anisotropic exchange interaction that has the same effect on the linewidth as the dipolar interaction. ${ }^{12}$

From the comparison of the intensity coming from the ESR data in Fig. 4, which is proportional to the susceptibility, with the magnetization obtained from SQUID data and shown in Fig. 2 we observe a large difference. For the first, the temperature variation is much more pronounced than for the latter. In this way, we also observe a large antiferromagnetic contribution comparing the ESR and magnetization data. In contrast with the magnetization measurements, the data coming from the ESR experiment show the contribution of the individual magnetic moments, irrespective of their interactions with neighbor moments. In this sense the ESR technique is a good one to allow an estimation of the 


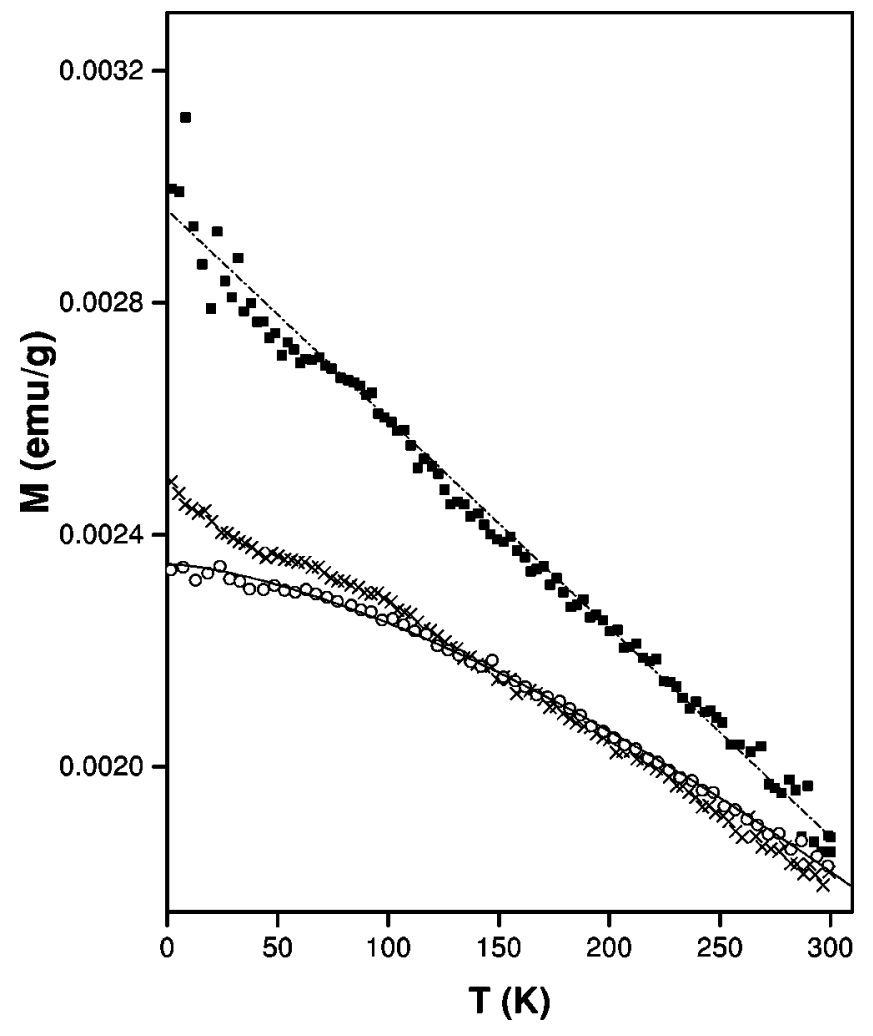

FIG. 5. Thermoremanence versus temperature of the sample cooled without a field from $300 \mathrm{~K}$ to $1.8 \mathrm{~K}$. At $1.8 \mathrm{~K}$ the field was applied for ten minutes and removed. (a) Open circles: $H$ $=500$ Oe. Solid line: fitting with $M(T)=M(0)-a T^{3 / 2}$. (b) Crosses: $H=1000$ Oe. (c) Squares: $H=5000$ Oe. Dash and dotted lines: fitting with $M(T)=M(0)-b T$.

number of spins per gram in the sample. This can be done comparing the area of the ESR absorption spectrum of a sample with known mass, with that of a standard with known number of spins 1/2, registered for the same experimental conditions. Since the linewidth of sample A at room temperature is around $4 \mathrm{G}$, the standard chosen was the Varian strong pitch with a linewidth of $4.24 \mathrm{G}$. The value obtained for sample A was $5.29 \times 10^{19} \mathrm{spins} / \mathrm{g}$. The uncertainty in this determination is around 15\% mainly because of coupling changes in the ESR cavity.

We can now make some estimations based on this result. First of all, we can tell, considering the $300 \mathrm{~K}$ ferromagnetic saturation value of $0.04 \mathrm{emu} / \mathrm{g}$, that $8.1 \%$ of the total number of spins contributes to the weak ferromagnetism. Although we have already shown in previous articles that this ferromagnetic behavior is intrinsic, such a high value is another evidence of it. From the fitting of Fig. 1 at $5 \mathrm{~K}$ we can write for the value of $\alpha$ :

$$
\alpha=\frac{\mu}{k_{B}\left(T-T_{C}\right)},
$$

where $\mu$ is the magnetic moment, $k_{B}$ the Boltzmann constant, and $T_{C}$ the critical temperature for the paramagneticferromagnetic transition. The saturation magnetization value at $5 \mathrm{~K}\left(M_{1}=0.0338 \mathrm{emu} / \mathrm{g}\right)$ obtained from the fitting, is

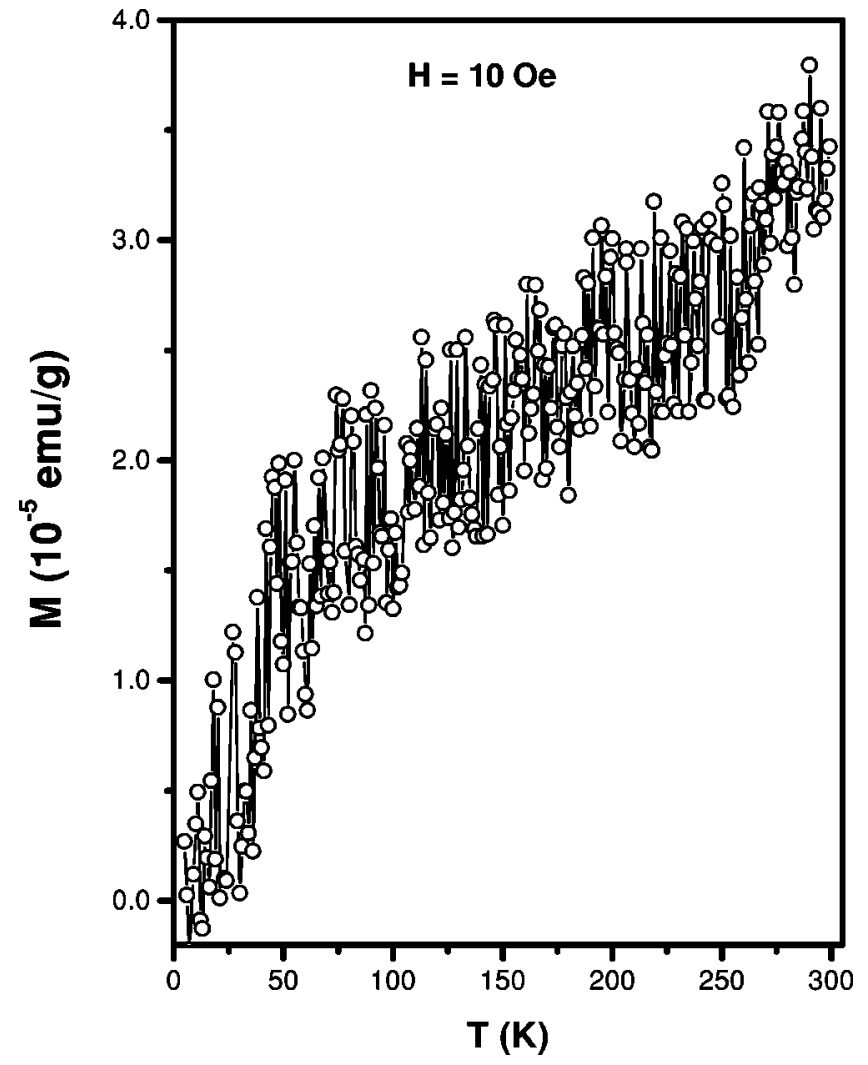

FIG. 6. Zero field cooling data showing magnetization versus temperature for sample D measured with $H=10$ Oe.

slightly smaller than the room temperature value (0.04 emu/ g). We can understand this result considering that at low temperatures the canting is smaller, probably because of freezing effects on the polymer chains. From the value of $M_{1}$ at $5 \mathrm{~K}$ we estimate that $6.8 \%$ of the total number of spins contributes for the weak ferromagnetic phase. This left a value of $0.93 \mu_{B}$ for the contribution coming from the metamagnetic component. This value can be obtained from the fitted value for $\alpha=1.68 \times 10^{-5} \mathrm{Oe}^{-1}$ if $T_{C}=1.28 \mathrm{~K}$. From the value of $M_{2}=0.256 \mathrm{emu} / \mathrm{g}$ we can find $2.97 \times 10^{19}$ spins/g if we assume a magnetic moment of $0.93 \mu_{B}$. We see that at $5 \mathrm{~K}$, around $43.8 \%$ of the magnetic moments are still coupling antiferromagnetically.

Based on the number of spins obtained from the ESR data the average doping level can be estimated. For poly(3methylthiophene) there is $6.13 \times 10^{21}$ monomers $/ g$. Assuming the width of a polaron of 6 monomeric units ${ }^{14}$ we find 1.02 $\times 10^{21}$ for the maximum number of polarons $/ g$. The value of $5.29 \times 10^{19}$ spins/g obtained from ESR corresponds to a $5.1 \%$ doping level. This value is in agreement with a doping level of $5 \%$ for a similar sample (sample E), estimated from electron dispersive spectroscopy (EDS) from the measurement of the $\mathrm{Cl} / \mathrm{S}$ ratio. We know that the doping level is not homogeneous due to the fact that conducting polymers are correlated insulators. ${ }^{15}$ However, we can make the assumption that the magnetic clusters are formed from polymeric chains of 10-100 monomeric units, with one polaronic defect for each polymeric chain. They are displaced in such a way that the doping anions are confined in a small region. The po- 
larons experience a superexchange interaction via the dopant anions. The partially crystalline low symmetry system allows a weak ferromagnetic behavior to arise due to DzialoshinskiMorya interaction among canted spins .

The complexity of the magnetic behavior in conducting polymers can also be observed from the thermoremanence data of Fig. 5. Sample $\mathrm{C}$ was cooled from room temperature to $1.8 \mathrm{~K}$ without an applied field. At $1.8 \mathrm{~K}$ a magnetic field was applied for ten minutes and then removed. The data was collected raising the temperature by $2 \mathrm{~K} / \mathrm{min}$. This experimental procedure was repeated for three different fields $H$ $=500 \mathrm{Oe}$ (open circles), $H=1000 \mathrm{Oe}$ (crosses), and $H$ $=5000$ Oe (squares). From the pure ferromagnetic behavior obtained from the thermoremance data we observe an increase in the magnetization with the increase of the magnetic field. Whereas for $500 \mathrm{Oe}$ we could fit the data with a classical tridimensional $M(T)=M(0)-a T^{3 / 2}$ behavior, ${ }^{16}$ as the field was increased a change toward a lower exponent can clearly be observed. At $H=5000$ Oe the data could be fitted with a bidimensional $M(T)=M(0)-b T$ behavior. The interesting point is that the critical temperature is approximately the same for both fields, around $815 \mathrm{~K}$. The change of dimensionality with the increase of magnetic field indicates that the magnetic field is also responsible for localizing the magnetic moments, i.e., they are confined in a plane perpendicular to the applied field. Localization induced by a large magnetic field has already been observed in conducting polymers ${ }^{17}$ which are responsible for promoting $M-I$ transitions.

In order to confirm the metamagnetic behavior observed from Figs. 1 and 2 we performed zero field cooling low field (10 Oe) measurements in a similar sample (sample D). The magnetization versus tempererature is shown in Fig. 6. In spite of the high dispersion observed from the data because of the very low values of magnetization, in the limit of the equipment sensibility, the antiferromagnetic behavior is now observed in the whole range of the temperatures. This is the antiferromagnetic state expected for low fields in a metamagnetic system. ${ }^{18}$

\section{CONCLUSIONS}

In this work we have studied the weak ferromagnetic phase in pressed pellets of $\mathrm{ClO}_{4}^{-}$partially doped poly(3methylthiophene) from magnetization and $X$-band ESR measurements. This weak ferromagnetic phase can clearly be observed at room temperature and the thermoremanence data allows an estimation of the critical temperature to be around $815 \mathrm{~K}$. In addition to the weak ferromagnetic behavior, metamagnetism is also observed in the system. This fact is not surprising because, since weak ferromagnets are a delicate balance of opposing forces, many of them are also metamagnetics. ${ }^{18}$

From the ESR and magnetization data we found that the room temperature weak ferromagnetic phase corresponds to $8.1 \%$ of the total number of spin $1 / 2$. Such a high value is again evidence of the intrinsic character of this weak ferromagnetic component. The doping level estimated from the ESR measurements is in good agreement with that estimated from EDS measurements, confirming the polaronic character of the magnetic behavior.

\section{ACKNOWLEDGMENTS}

This work was partially supported by Fundação de Amparo à Pesquisa do Estado de São Paulo e Conselho Nacional de Desenvolvimento Científico e Tecnológico.
${ }^{1}$ H. Shirakawa, E.J. Louis, A.G. MacDiarmid, C.K. Chiang, and A.J. Heeger, J. Chem. Soc. Chem. Commun. 579 (1977) .

${ }^{2}$ C.K. Chiang, C.R. Fischer, Y.W. Park, A.J. Heeger, H. Shirakawa, E.J. Louis, S.C. Gau, and A.G. MacDiarmid, Phys. Rev. Lett. 39, 1098 (1977).

${ }^{3}$ H. Fukutome, A. Takahashi, and M. Ozaki, Chem. Phys. Lett. 133, 34 (1987).

${ }^{4}$ A.M.S. Macedo, M.C. dos Santos, M.D. Coutinho-Filho, and C.A. Macedo, Phys. Rev. Lett. 74, 1851 (1995).

${ }^{5}$ K. Yoshizawa, K. Tanaka, T. Yamato, and J. Yamauchi, J. Chem. Phys. 96, 5516 (1992).

${ }^{6}$ J.N. Devine, J.A. Crayston, and J.C. Walton, Synth. Met. 103, 2294 (1999).

${ }^{7}$ A. Rajca, J. Wongsriratanakul, and S. Rajca, Science 294, 1503 (2001).

${ }^{8}$ A.A. Correa, L. Walmsley, L.O.S. Bulhões, W.A. Ortiz, A.J.A. de Oliveira, and E.C. Pereira, Synth. Met. 121, 1836 (2001).
${ }^{9}$ E.C. Pereira, A.A. Correa, L.O.S. Bulhões, P.C. Aleixo, J.A. Nóbrega, A.J.A. de Oliveira, W.A. Ortiz, and L. Walmsley. J. Magn. Magn. Mater. 226, 2023 (2001).

${ }^{10}$ T. Morya, in Weak Ferromagnetism, Vol. 1 of Magnetism, edited by G.T. Rado and H. Suhl (Academic Press, London, 1963).

${ }^{11}$ E. Stryjewski and N. Giordano, Adv. Phys. 26, 487 (1977).

${ }^{12}$ A. Bencini and D. Gatteschi, Electron Paramagnetic Resonance of Exchange Coupled Systems (Springer-Verlag, Berlin, 1990).

${ }^{13}$ N. F. Mott, Metal-Insulator Transitions (Barnes \& Noble, New York, 1974), Chap. 2.

${ }^{14}$ D. Bertho and C. Jouanin, Phys. Rev. B 35, 626 (1987).

${ }^{15}$ S. A. Kivelson and V. J. Emery, Synth. Met. 65, 249 (1994).

${ }^{16}$ C. Kittel, Introduction to Solid State Physics (Wiley, New York, 1995).

${ }^{17}$ A.J. Heeger, J. Supercond. 14, 261 (2001).

${ }^{18}$ C.M. Hurd, Contemp. Phys. 23, 469 (1982). 